and (16) gives

$$
\begin{aligned}
\frac{5}{24}\left(Y_{4}^{4,4}+Y_{4}^{4,-4}+Y_{4}^{-4,4}\right. & \left.+Y_{4}^{-4,-4}\right) \\
& +\left(\frac{35}{288}\right)^{1 / 2}\left(Y_{4}^{4,0}+Y_{4}^{-4,0}+Y_{4}^{0.4}+Y_{4}^{0,-4}\right)+\frac{7}{12} Y_{4}^{0,0}
\end{aligned}
$$

as a properly invariant function.

The author wishes to extend his appreciation to Professor R. Bersohn, without whose assistance this work would have been impossible.

\title{
ON THE MOTION OF A SIMPLE PENDULUM*
}

\section{BY BORIS GARFINKEL (Ballistic Research Laboratories, Aberdeen Proving Ground, Md.)}

Abstract. The vanishing of the tension in a simple pendulum supported by a flexible cord causes the particle to pass from the circular to a parabolic trajectory. The number and the nature of such transitions are related here to the value of the initial energy.

1. When the initial energy of a simple pendulum lies in a certain interval, the tension vanishes at some instant of the motion. Then, if the support is provided by a flexible cord, the particle passes from the circular to a parabolic trajectory. The number and the nature of such transitions are shown here to be precisely related to a dimensionless energy parameter, $\xi$. Despite the intrinsic interest and the relative simplicity of this motion, it does not appear to have been treated in the literature.

Let $l$ be the length, $m$ the mass of the pendulum, $r$ its distance from the point of support, $\theta$ the angular coordinate measured from the downward-drawn vertical line, and $g$ the acceleration of gravity. The constraint

$$
l-r \geq 0
$$

can be replaced by the condition

$$
\lambda(l-r)=0,
$$

where $\lambda$ is a multiplier vanishing if $l>r$ and admitting a non-zero value if $l=r$. The Lagrangian of the system,

$$
L=\frac{1}{2} m\left(r^{\cdot 2}+r^{2} \theta^{\cdot 2}\right)+m g r \cos \theta+\lambda(l-r),
$$

leads to the differential equations of motion,

$$
m\left(r^{\cdot}-r \theta^{\cdot 2}-g \cos \theta\right)+\lambda=0, \quad r \theta^{\cdot}+2 r^{\cdot} \theta^{\cdot}+g \sin \theta=0,
$$

which together with (2) and (1) determine the functions $r(t), \theta(t), \lambda(t)$ when the initial conditions are prescribed. From (4.1) the multiplier $\lambda(t)$ can be identified with the

*Received July 22, 1957. 
tension of the cord. Let the initial conditions now be:

$$
\begin{array}{ll}
r(0)=l, & \theta(0)=0, \\
r^{\cdot}(0)=o, & \theta^{\cdot}(0)=\left(2 E / m l^{2}\right)^{1 / 2},
\end{array}
$$

$E$ being the initial energy measured from the lowest point. Then the particle moves in a circular arc $r(t)=l$ until $\lambda$ vanishes. The integrals of the motion, deduced from (4), are

$\left(\theta^{\circ} / \omega\right)^{2}-2 \cos \theta=2\left(2 c^{2}-1\right)=3 \xi, \quad \sin (\theta / 2)=\min (1, c) \operatorname{sn}[\omega t / \max (1, c)]$,

where the constants $\omega, c, \xi$ are defined by

$$
\omega^{2}=g / l, \quad c^{2}=E / 2 m g l, \quad \xi=\frac{2}{3}\left(2 c^{2}-1\right),
$$

and the modulus of the elliptic function sn is

$$
\kappa=\min (c, 1 / c) \text {. }
$$

The dimensionless parameter $\xi$ is seen from ( 7 ) to be proportional to the energy measured from the horizontal configuration $r=l, \theta=\pi / 2$; its range is $-2 / 3 \leq \xi<\infty$.

2. At the instant $t=t_{1}$ of the vanishing of the tension the coordinates and their derivatives, obtained from (4.1) and (6.1) with the substitutions $\lambda=0, r=l, r^{*}=0$, are given by

$$
\begin{aligned}
\cos \theta_{1}=-\xi, & r_{1}=l, \\
\theta_{1} / \omega=\xi^{1 / 2}, & r_{1}^{\cdot}=0 .
\end{aligned}
$$

Hence $\theta_{1}$ and $\theta_{\mathrm{i}}$ are real if and only if $\xi$ lies in the range

$$
0 \leq \xi \leq 1,
$$

and $\pi / 2 \leq \theta_{1} \leq \pi, 0 \leq \theta_{1}^{\circ} \leq \omega$. At $t=t_{1}$ there occurs a transition from the circular to a parabolic trajectory. The latter corresponds to the solution of (4) with $\lambda=0, r \leq l$, and the initial conditions (9), and is represented by the equations

$$
\begin{aligned}
(r / l)^{2} & =1-(\omega \tau)^{3}\left[\xi\left(1-\xi^{2}\right)\right]^{1 / 2}-(\omega \tau)^{4} / 4, \\
r \sin \theta / l & =\left(1-\xi^{2}\right)^{1 / 2}-\xi^{3 / 2}(\omega \tau),
\end{aligned}
$$

where

$$
\tau=t-t_{1} .
$$

When the particle re-enters the circle, $r$ again assumes the value $r=l$, and (11) yields

$$
\begin{aligned}
\omega \tau & =4\left[\xi\left(1-\xi^{2}\right)\right]^{1 / 2}, \\
\theta_{2} & =2 \pi-3 \theta_{1}, \quad r_{2}=l \\
\theta_{2}^{\cdot} & =\theta_{1}\left(-3+12 \xi^{2}-8 \xi^{4}\right), \\
r_{2}^{*} & =8 l \omega\left[\xi\left(1-\xi^{2}\right)\right]^{3 / 2} .
\end{aligned}
$$

Such an alternation of the trajectory between the circle and a parabola we shall, for the sake of brevity, designate by the term "flip." At the instant $t=t_{2}$ of re-entry of the circle the ideal cord, assumed to be inextensible and infinitely strong, enforces the 
constraint (1) by generating an impulse equal and opposite to the radial momentum $m$. While the latter is being annihilated, the angular momentum $m r^{2} \theta^{\circ}$ is conserved, and the energy is thus diminished by the quantity $\mathrm{mr}^{\cdot 2} / 2$. The corresponding diminution of $\xi$ can be calculated from (13.4) and the definitions (7); the new value $\xi^{\prime}$ can then be written as a function, $f(\xi)$, in the form

$$
\xi^{\prime}=f(\xi)=\xi-\frac{64}{3}\left[\xi\left(1-\xi^{2}\right)\right]^{3}, \text { for } 0 \leq \xi \leq 1 .
$$

Of course, if $\xi$ lies outside the range (10) flips do not occur and the energy is conserved. Therefore

$$
f(\xi)=\xi, \text { for } \xi(1-\xi)<0 .
$$

In particular, $-2 / 3 \leq \xi<0$ and $\xi>1$ correspond to the states of oscillation and circulation respectively.

A few curious details of the motion will now be summarized.

1. "Oscillatory" flips, for which the sense of $\theta_{2}^{*}$ is opposite to that of $\theta_{1}^{*}$, occur if $0<\xi<\frac{1}{2}\left[3-3^{1 / 2}\right]^{1 / 2}=0.56$; "circulatory" flips occur if $0.56 \leq \xi<1$. The separating value corresponds to $\theta_{2}^{*}=0$.

2. The maximum energy loss per flip corresponds to $\xi=3^{-1 / 2}=0.57$, with the parabola passing through the point of suspension.

3. The "amplitude" of a flip can be defined as $\left.\alpha=\mid \sin \left[\left(\theta_{1}-\theta_{2}\right) / 2\right]\right)$. Its maximum $\alpha=1$, corresponds to $\xi=2^{-1 / 2}=0.71, \theta_{1}=3 \pi / 4, \theta_{2}=\pi / 4$; its minimum, $\alpha=0$, occurs when $\xi=0, \theta_{1}=\theta_{2}=\pi / 2$ and when $\xi=1, \theta_{1}=\theta_{2}=\pi$.

4. The entire history of the motion can now be described in terms of the function $f(x)$, which is graphed and tabulated below, together with the corresponding values of $\theta_{1}$ and $\theta_{2}$.

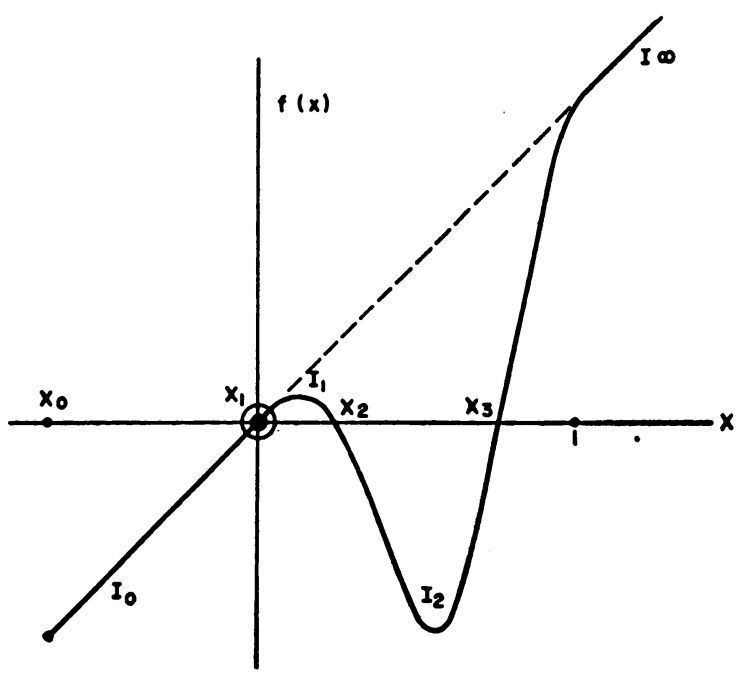

FIG. 1. Graph of the function $f(x)$. 
TABLE 1

Function $f(x), 0 \leq x \leq 1$

\begin{tabular}{c|r|r|r}
\hline$x$ & \multicolumn{1}{|c|}{$f(x)$} & \multicolumn{1}{|c}{$\theta_{1}$} & \multicolumn{1}{c}{$\theta_{2}$} \\
\hline 0.0 & 0.000 & $90^{\circ} .0$ & $90^{\circ} .0$ \\
0.1 & 0.079 & 95.7 & 72.8 \\
0.2 & 0.049 & 101.5 & 55.4 \\
0.3 & -0.134 & 107.5 & 37.6 \\
0.4 & -0.409 & 113.6 & 19.3 \\
0.5 & -0.625 & 120.0 & 0.0 \\
0.6 & -0.608 & 126.9 & -20.6 \\
0.7 & -0.271 & 134.4 & -43.3 \\
0.8 & 0.290 & 143.1 & -69.4 \\
0.9 & 0.793 & 154.2 & -102.5 \\
1.0 & 1.000 & 180.0 & -180.0 \\
\hline
\end{tabular}

Some of the important properties of $f(x)$ are listed below:

1. $f(x)$ is of class $C^{2}$; i.e. it has continuous derivatives up to the second order in its entire domain $-2 / 3 \leq \xi<\infty$.

2. The stationary values of $f(x)$ are given by $\min f(x)=-0.655=f(0.544), \max$ $f(x)=+0.086=f(0.130)$.

3. The real zeros of $f(x)$ are:

$$
x_{1}=0, \quad x_{2}=0.236, \quad x_{3}=0.751 .
$$

4. These zeros, together with the boundary point $x_{0}=-2 / 3$ and the recursive relation

$$
f\left(x_{k+4}\right)=x_{k+2}, \quad k=0,1, \cdots,
$$

define a monotone increasing sequence $\left\{x_{k}\right\} ; k=0,1, \ldots$, converging to $x_{\infty}=1$. This result follows from the facts that $x_{2}$ and $x_{3}$ lie in the interval $\max f<x<1$, that in this interval the inverse function $f^{-1}(x)$ is defined and is bounded by the inequality $x<f^{-1}(x)<1$. The first nine terms of the sequence are:

$-0.667,0.000,0.236,0.751,0.791,0.889,0.899,0.930,0.935, \cdots$,

The value $\xi$ lies in some interval $I_{k}$, such that

$$
\begin{array}{lll}
x_{k} \leq \xi<x_{k+1} & \text { if } & -2 / 3 \leq \xi<1, \\
k=\infty & \text { if } & 1 \leq \xi<\infty .
\end{array}
$$

The three intervals $k=0, k=1, k=\infty$ are the only stable states of $\xi$, in the sense that the system once in such a state will remain in that state forever. In particular, the two extreme states, $k=0,-2 / 3 \leq \xi<0$, and $k=\infty, 1 \leq \xi<\infty$ are the classical oscillation and circulation respectively. The state $k=1,0 \leq \xi<0.236$ contains an infinite succession of oscillatory flips whose amplitude converges to zero; the motion converges to an oscillation with $\xi=0, \max \theta=\pi / 2$. In all other states a flip results in the jump $\Delta k=-2$ in the step-function $k(t)$. Consequently the particle executes 
$[k(0) / 2]$ flips before descending to the state $k=0$ if $k(0)$ is even or to the state $k=1$ if $k(0)$ is odd. The bracket above denotes the integral part of a number. The total number $N$ of flips and the ultimate state $k(\infty)$ of the system are hence given by the following table.

TABLE 2

History of the motion

\begin{tabular}{c|c|cl}
\hline$k(0)$ & $N$ & $k(\infty)$ & Cltimate state \\
\hline $\begin{array}{c}\text { even } \\
\text { odd }\end{array}$ & {$[k(0) / 2]$} & 0 & oscillation \\
$\infty$ & $\infty$ & 1 & flipping \\
& 0 & $\infty$ & circulation \\
\hline
\end{tabular}

As an example, consider $\xi(0)=0.777$. From inspection of the sequence (17) it is seen that $k(0)=3$. After one flip the system reaches the state $k=1$, characterized by an infinite succession of flips, as indicated in Table 2 . It is to be observed that if the support were rigid the pendulum would circulate, since $\xi$ exceeds the critical value $\xi=2 / 3,(c=1)$, which separates the oscillatory and the circulatory states in the classical case.

4. Summary. In the case of a simple pendulum supported by a flexible cord, whenever the tension vanishes the particle passes from the circular to a parabolic trajectory. The energy loss occurring upon re-entry into the circle is given by the expression

$$
\begin{aligned}
f(x)-x & =-\frac{64}{3}\left[x\left(1-x^{2}\right)\right]^{3}, \quad(0<x<1) \\
& =0, \quad(x(1-x)<0),
\end{aligned}
$$

where $x$ and $f(x)$ are the old and the new values of the energy, measured from the horizontal configuration $\theta=\pi / 2$, and normalized by a divisor $3 \mathrm{mgl} / 2$. The state of the pendulum is characterized by an integer $k$, such that

$$
\begin{aligned}
x_{k} & \leq x<x_{k+1}, & & (x<1), \\
k & =\infty, & & (x \geq 1),
\end{aligned}
$$

where the sequence $\left\{x_{k}\right\} ; k=0,1, \cdots$, is monotone increasing, converging to $x_{\infty}=1$, and defined by

$$
\begin{gathered}
f\left(x_{k+4}\right)=x_{k+2}, \quad k=0,1, \cdots \\
x_{0}=-\frac{2}{3}, \quad x_{1}=0, \quad x_{2}=0.236, \quad x_{3}=0.751 .
\end{gathered}
$$

Here $x_{1}, x_{2}$, and $x_{3}$ are the three real zeros of $f(x)$, and $x_{0}$ is the lowest possible energy. The two extreme states $k=0$ and $k=\infty$ correspond respectively to pure oscillation and pure circulation. The state $k=1$, which does not arise in the usual case of a rigid support, contains an infinite succession of parabola-circle transitions. The three states $k=0, k=1$ and $k=\infty$ are the only stable states, in the sense that the system once in such a state will remain in that state forever. From an unstable state the system will ultimately descend to the state $k=0$ if $k(0)$ is even, or to the state $k=1$ if $k(0)$ is odd. In this process there occurs a finite number of parabola-circle transitions, this number being equal to $[k(0) / 2]$, the largest integer not exceeding $k(0) / 2$. 